Pak. j. sci. ind. res. Ser. A: phys. sci. 2020 63A(1) 1-11

\title{
Synthesis of New Substituted Tetrazole and 4-Thiazolidinone from Schiff's Bases
}

\author{
Amena Alyas Ahmed*, Natiq Ghanim Ahmed and Ahmad Khuder Ahmad \\ Department of Chemistry, College of Education for Pure Science, University of Mosul, Mosul, Iraq
}

(received November 11, 2018; revised May 2, 2019; accepted May 9, 2019)

\begin{abstract}
The present work involved the synthesis of compound (1) (1-amino-4-methyl-6-phenyl pyrimidine$2-(1 \mathrm{H})$-thione). This compound reacts with different aromatic aldehyde using glacial acetic acid as catalytic on absolute ethanol to give a new series of Schiff's bases (2-7). New thiazolidine-4-one were prepared from reactions of Schiff's bases $(\mathbf{2 , 3 , 4 , 7 )}$ with thioglycolic acid in absolute ethanol giving compounds (8-11). Finally the preparation of new tetrazole derivatives $(\mathbf{1 2 - 1 5})$ by reaction of Schiff's bases $(\mathbf{2 , 3}, \mathbf{4 , 7})$ with sodium azide in THF. The structure of the synthesized compounds are confirmed by I.R., ${ }^{1} \mathrm{H}-\mathrm{NMR}$ and ${ }^{13} \mathrm{C}-\mathrm{NMR}$ spectra and some physical data.
\end{abstract}

Keywords: tetrazole, thiazolidinone, schiff's bases

\section{Introduction}

Schiff bases are used as substrates in the preparation of number of industrial and biologically active compounds via ring closure, (cyclo addition and replacement reaction) Harika et al. (2014). Thiazolidinone derivatives have various pharmacological activities such as antibacterial (Subdhi et al., 2005), antifungal (Patel, 2011), anticancer (Srivustava et al., 2002), anticonvulsant (Parekh et al., 2004) and herbicidal actions (Qien et al., 2006). Tetrazoles have been found to exhibit antihistamine (Samadhiya and Halve, 2001), antifungal properties (Pradip and Berad, 2008).

\section{Materials and Methods}

All reagents and chemicals are from BDH and Fluka, used without purification. Melting points were measured using: electro thermal melting points apparatus type (not corrected). FT spectra were recorded on Shimadzu FT IR-8400 Infrared Spectrophotometer. ${ }^{1} \mathrm{H}-\mathrm{NMR}$ and ${ }^{13} \mathrm{C}$-NMR spectra were recorded by Geo. 1400(400 $\mathrm{MHz}$ ) using acetone $\mathrm{d}^{6}$ and $\mathrm{CDCl}_{3}$ as solvent in UK Loughborough.

Synthesis of 1-amino-4-methyl-6-phenyl pyrimidine2-(1H) thion (1). A mixture of ( 0.01 mole) of benzoyl acetone and $(0.01 \mathrm{~mole})$ of thiosemicarbazide in (50 $\mathrm{mL}$ ) absolute ethanol containing 3 drops of piperidine, was refluxed for $5 \mathrm{~h}$. The solvent was then removed and the resulting solid was recrystallized from ethanol reported in (Moayed, 2017).

*Author for correspondence; E-mail: altaeeamena@gmail.com
Synthesis of schiff bases (2-7). A mixture of compound (1) $(0.01$ mole) and different aromatic aldehydes (2nitro benzaldehyde, 3-nitro benzaldehyde, 4-nitro benzaldehyde, 4-amino benzaldehyde, 4-methoxy benzaldehyde, 4-phthaladehyde ( 0.01 mole) in absolute ethanol $(25 \mathrm{~mL})$ containing 3 drops of glacial acetic acid was stirring for $4 \mathrm{~h}$. The solvent was evaporated under vaccum, the yielded solid crystallized from methanol (Al-Gwady et al, 2018; Natiq and Hussein, 2016). The physical properties are listed in Table 1.

Synthesis of thiazolidinones derivatives (8-11). Mercaptoacetic acid ( 0.002 mole) in absolute ethanol $(10 \mathrm{~mL})$ was added slowly to $(0.001 \mathrm{~mole})$ of Schiff bases $(\mathbf{2 , 3}, \mathbf{4}, \mathbf{7})$. The mixture was refluxed for $5 \mathrm{~h}$. Excess solvent was evaporated and the residue was treated with potassium bicarbonate to produce compounds (AlMosawi, 2014; Lakum et al., 2014; Hussain et al., 2014). The solid precipitates were recrystallized from ethanol. The physical properties are listed in Table 1.

Synthesis of tetrazole derivatives (12-15). The mixture of compound $(\mathbf{2 , 3 , 4 , 7 )} \quad(0.0004$ mole) dissolved in $(20 \mathrm{~mL})$ tetrahydrofuran and $(0.0006$ mole) sodium azide was refluxed for $16 \mathrm{~h}$. The precipitate was filtered and recrystallized from absolute ethanol (Mahmoud et al., 2013). The physical properties are listed in Table 1.

\section{Results and Discussion}

The new Schiff's bases were synthesized from the reaction of 1-amino-4-methyl-6-phenyl pyrimidine-2$\left({ }^{1} \mathrm{H}\right)$-thione (1) with different aromatic aldehyde in 
Table 1. Some physical properties of the compounds (1-15)

\begin{tabular}{llllc}
\hline \hline $\begin{array}{l}\text { Comp. } \\
\text { no. }\end{array}$ & Structure and Name & $\begin{array}{l}\text { Molecular formula } \\
\& \mathrm{M} . \mathrm{W} .\end{array}$ & $\begin{array}{l}\text { M.P. }{ }^{\circ} \mathrm{C} \\
\text { \& colour }\end{array}$ & Yield (\%) \\
\hline 1 & & $\begin{array}{l}\mathrm{C}_{11} \mathrm{H}_{14} \mathrm{~N}_{4} \mathrm{O}_{2} \mathrm{~S} \\
217\end{array}$ & $\begin{array}{l}160-162 \\
\text { Pale yellow }\end{array}$ & 95 \\
& & & & \\
\end{tabular}

1-Amino-4-methyl-6-phenyl pyrimidine-2-(1H)-thione

2<smiles>Cc1cc(-c2ccccc2)n(NCc2ccccc2[N+](=O)[O-])c(=S)n1</smiles>

$\mathrm{C}_{18} \mathrm{H}_{14} \mathrm{~N}_{4} \mathrm{O}_{2} \mathrm{~S}$ 350
228-230

White

74

(1)

4-methyl-1-\{[1E).(2-nitrophenyl) methylene]amino\}-6-phenyl pyrimidine-2(1H)-thione

3<smiles>Cc1cc(-c2ccccc2)n(/N=C/c2cccc([N+](=O)[O-])c2)c(=S)n1</smiles>

$\mathrm{C}_{18} \mathrm{H}_{14} \mathrm{~N}_{4} \mathrm{O}_{2} \mathrm{~S}$ 350 189-190

White
75

83

238-240

Yellow

5<smiles>Cc1cc(-c2ccccc2)n(/N=C/c2ccc(N)cc2)c(=S)n1</smiles>

$\mathrm{C}_{18} \mathrm{H}_{16} \mathrm{~N}_{4} \mathrm{~S}$

320

Pale yellow

4-methyl-1-\{[(1E).(4-amino phenyl)methylene]amino\}-6-phenyl pyrimidine-2(1H)-thione 
6<smiles>COc1ccc(C=Nn2c(-c3ccccc3)cc(C)nc2=S)cc1</smiles>

148-150

Light brown
51 335

$\mathrm{C}_{19} \mathrm{H}_{17} \mathrm{~N}_{3} \mathrm{OS}$

65

324-326

White

65<smiles>Cc1cc(-c2ccccc2)n(/N=C/c2ccc(/C=N/n3c(-c4ccccc4)ccnc3=O)cc2)c(=S)n1</smiles>

1,1'-((1,4-phenylenebis(methaneylylidene))bis(azaneylylidene))bis(4-methyl-6-phenylpyrimidine-2(1H)-thione)

8

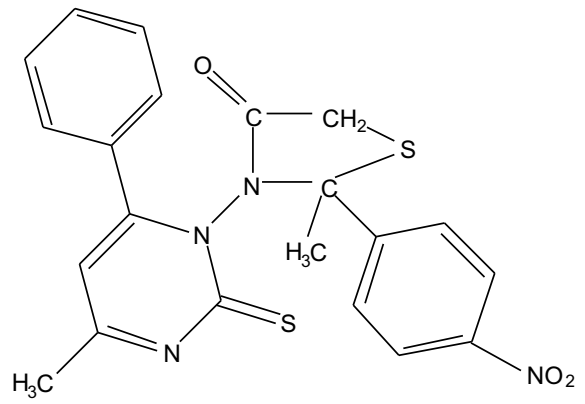

$\mathrm{C}_{20} \mathrm{H}_{16} \mathrm{~N}_{4} \mathrm{O}_{3} \mathrm{~S}_{2}$ 424

$181-183$

Green
62

(1)

3-(4-methyl-6-phenyl-2-thioxo pyrimidin-1(2H)-yl)-2-(2-nitrophenyl)-1,3-thiazolidin-4-one

9

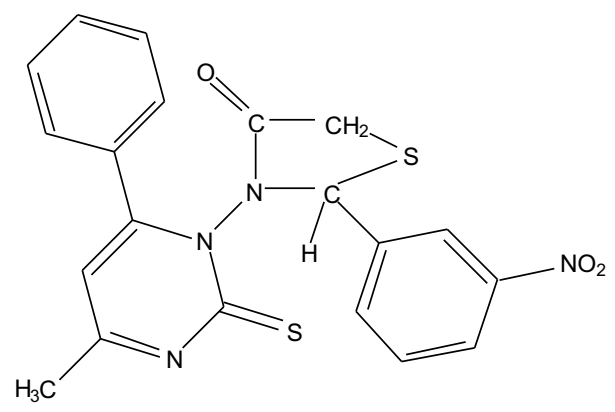

$\mathrm{C}_{20} \mathrm{H}_{16} \mathrm{~N}_{4} \mathrm{O}_{3} \mathrm{~S}_{2}$ 424
186-188

White

61 
10

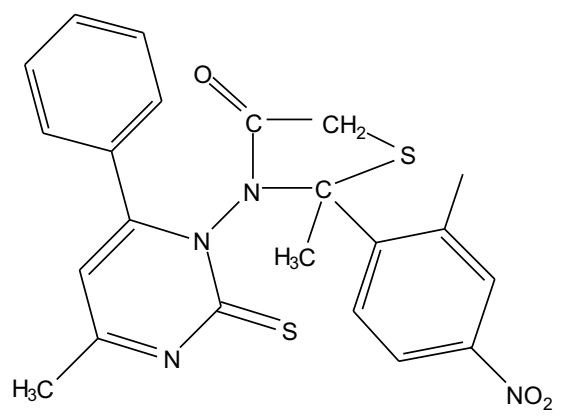

$\mathrm{C}_{20} \mathrm{H}_{16} \mathrm{~N}_{4} \mathrm{O}_{3} \mathrm{~S}_{2}$ 424

244-246

Yellow
86

3-(4-methyl-6-phenyl-2-thioxo pyrimidin-1(2H)-yl)-2-(4-nitrophenyl)-1,3-thiazolidin-4-one

11

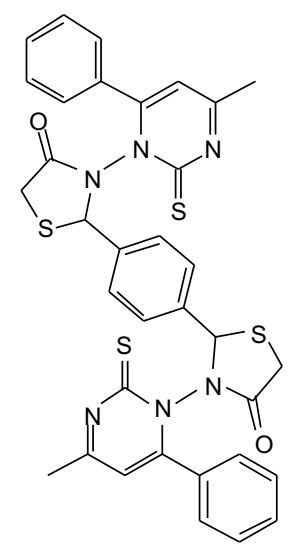

$\mathrm{C}_{34} \mathrm{H}_{28} \mathrm{~N}_{6} \mathrm{O}_{2} \mathrm{~S}_{4}$ 681
318-320

White
71

(1)

2,2'-(1,4-phenylene)bis(3-(4-methyl-6-phenyl-2-thioxopyrimidin-1(2H)-yl)thiazolidin-4-one)

12<smiles>Cc1cc(-c2ccccc2[N+](=O)[O-])n(N2NN=NC2c2ccccc2)c(=S)n1</smiles>

$\mathrm{C}_{18} \mathrm{H}_{15} \mathrm{~N}_{7} \mathrm{O}_{2} \mathrm{~S}$ 393
200-202

Light brown
51

4-methyl-1-[5-(2-nitro phenyl)-2,5-dihydro-1H-tetrazol-1-yl]-6-phyenyl pyrimidine-2(1H)-thione

13<smiles>Cc1cc(-c2cccc([N+](=O)[O-])c2)n(N2NN=NC2c2ccccc2)c(=S)n1</smiles>

$\mathrm{C}_{18} \mathrm{H}_{15} \mathrm{~N}_{7} \mathrm{O}_{2} \mathrm{~S}$ 393
228-230

Brown

4-methyl-1-[5-(3-nitro phenyl)-2,5-dihydro-1H-tetrazol-1-yl]-6-phyenyl pyrimidine-2(1H)-thione 
14<smiles>Cc1cc(-c2ccccc2)n(N2NN=NC2c2ccc([N+](=O)[O-])cc2)c(=S)n1</smiles>

$\mathrm{C}_{18} \mathrm{H}_{15} \mathrm{~N}_{7} \mathrm{O}_{2} \mathrm{~S}$ 393
250-252

Yellow 75
15

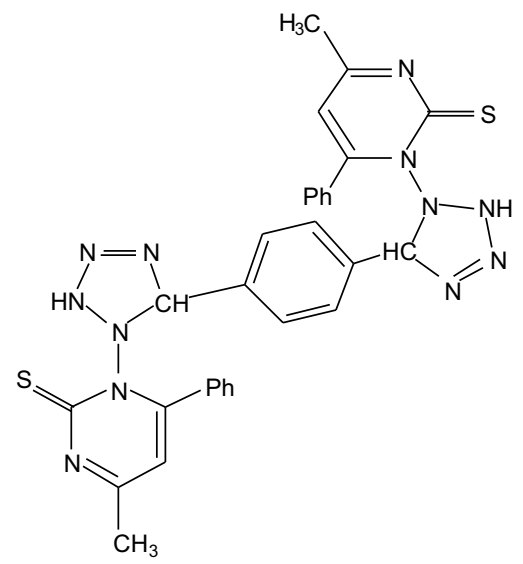

$\mathrm{C}_{30} \mathrm{H}_{26} \mathrm{~N}_{122} \mathrm{~S}_{2}$ 618.74
268-270

Yellow
69

,1'-(1,4-phenylenebis(2,5-dihydro-1H-tetrazole-5,1-diyl))bis(4-methyl-6-phenylpyrimidine-2(1H)-thione)

absolute ethanol and in the catalytic amount of glacial acetic acid. The FT-IR spectra of Schiff's bases (2-7) showed the absence of peak of carbonyl groups and the new peaks which appeared at 1580-1606 $\mathrm{cm}^{-1}$ which are attributed to the new azomethine $(\mathrm{C}=\mathrm{N})$ group (Dhanya et al., 2014). Some spectral data are listed in Table 2. Thiazolidinoe compounds (8-11) were prepared from the reaction of Schiff's bases $(\mathbf{2}, \mathbf{3}, \mathbf{4 , 7})$ with thioglycolic acid in absolute ethanol. FT-IR spectrum showed sharp peaks at $(1724-1700) \mathrm{cm}^{-1}$ due to $(\mathrm{C}=\mathrm{O})$ imide stretching frequency, a good evidence for the success of this step of reaction (Kumar et al., 2012). Some spectral data are listed in Table 2. Tetrazole compound (12-15) were synthesized from the reaction of Schiff's bases $(\mathbf{2}, \mathbf{3}, \mathbf{4 , 7})$ with sodium azide in THF. The FT-IR absorption bands disappearance at (1580$1599) \mathrm{cm}^{-1}$ is give good evidence for the success step of reaction. These absorption bands due to $(\mathrm{C}=\mathrm{N})$ imine group stretching frequency (Saad , 2018). Also FT-IR spectra of tetrazole showed clear absorption bands at (1441-1499) $\mathrm{cm}^{-1}$ due to $(\mathrm{N}=\mathrm{N})$. Beside this, the FT-IR spectra were appeared of band at (2077-2360) $\mathrm{cm}^{-1}$ attributed to the stretching frequency of azide group (Majeed and Saoud, 2013). Some spectral data are listed in Table 2.

The ${ }^{1} \mathrm{H}$ NMR \& ${ }^{13} \mathrm{C}$ NMR spectrum showed the following bands. Compound (1). ${ }^{1} \mathrm{H} \mathrm{NMR}\left(\mathrm{CDCl}_{3}\right.$, $400 \mathrm{MHz}): \delta=7.30-7.37(\mathrm{~m}, 5 \mathrm{H}, \mathrm{Ar}-\mathrm{H}), 5.96\left(\mathrm{~s},{ }^{1} \mathrm{H}\right.$, $\mathrm{H}-\mathrm{C}=\mathrm{C}), 3.45-3.40\left(\mathrm{dd}, 2 \mathrm{H}, \mathrm{NH}_{2}\right), 2.04\left(\mathrm{~s}, 3 \mathrm{H}, \mathrm{CH}_{3}\right)$. ${ }^{13} \mathrm{C} \mathrm{NMR}\left(\mathrm{CDCl}_{3}, 400 \mathrm{MHz}\right): \delta=175.5(\mathrm{C}=\mathrm{S}), 155.4$ $(\mathrm{C}=\mathrm{N}), 144.1,128.7,128.0,124.0,95.3(\mathrm{Ar}-\mathrm{H}), 55.2$ $(\mathrm{C}=\mathrm{C}-\mathrm{H}), 16.1\left(\mathrm{CH}_{3}\right)$ (Selvam et al., 2012).

Compound (2). ${ }^{1} \mathrm{H}$ NMR (acetone- $\left.\mathrm{d}^{6}, 400 \mathrm{MHz}\right): \delta=$ 8.61 (s,1H, H-C=N), 8.5-8.1 (m, 4H, Ar-H), 7.5-7.7 (m, $5 \mathrm{H}, \mathrm{Ar}-\mathrm{H}), 6.4(\mathrm{~s}, 1 \mathrm{H}, \mathrm{H}-\mathrm{C}=\mathrm{C}) .2 .8\left(\mathrm{~s}, 3 \mathrm{H}, \mathrm{CH}_{3}\right) .{ }^{13} \mathrm{C}$ NMR (acetone $\left.-\mathrm{d}^{6}, 400 \mathrm{MHz}\right): \delta=180.2(\mathrm{C}=\mathrm{S}), 148.9$ $(\mathrm{C}=\mathrm{N}), 140.0,136.4,133.2,130.1,121.3(\mathrm{Ar}-\mathrm{H}), 28.4$ $\left(\mathrm{CH}_{3}\right)$ (Dhanya et al., 2014).

Compound (4). ${ }^{1} \mathrm{H}$ NMR (DMSO-d 6 , $\left.400 \mathrm{MHz}\right): \delta=$ $8.4(\mathrm{~s}, 1 \mathrm{H}, \mathrm{H}-\mathrm{C}=\mathrm{N}), 8.2-8.1(\mathrm{~m}, 4 \mathrm{H}, \mathrm{Ar}-\mathrm{H}), 7.4-7.3(\mathrm{~m}$, $4 \mathrm{H}, \mathrm{Ar}-\mathrm{H}), 6.4(\mathrm{~s}, 1 \mathrm{H}, \mathrm{H}-\mathrm{C}=\mathrm{C}) .3 .3\left(\mathrm{~s}, 3 \mathrm{H}, \mathrm{CH}_{3}\right) .{ }^{13} \mathrm{C}$ NMR (DMSO-d $\left.{ }^{6}, 400 \mathrm{MHz}\right): \delta=179.9(\mathrm{C}=\mathrm{S}), 148.1$ $(\mathrm{C}=\mathrm{N}), 142.1,140.0,129.8,125.5$, (phenyl ring), 39.5 $\left(\mathrm{CH}_{3}\right)$ (Dhanya et al., 2014). 
Compound (5). ${ }^{1} \mathrm{H}$ NMR (DMSO-d $\left.\mathrm{d}^{6}, 400 \mathrm{MHz}\right): \delta=$ $10.7\left(\mathrm{~s}, 2 \mathrm{H}, 2 \mathrm{NH}_{2}\right), 7.8(\mathrm{~s}, 1 \mathrm{H}, \mathrm{H}-\mathrm{C}=\mathrm{N}), 6.8-7.31(\mathrm{~m}$, 4H, Ar-H), 7.38-7.46 (m, 5H, Ar- H), 1.67 (s, 3H, $\left.\mathrm{CH}_{3}\right)$. ${ }^{13} \mathrm{C}$ NMR (DMSO-d $\left.{ }^{6}, 400 \mathrm{MHz}\right): \delta=179.7(\mathrm{C}=\mathrm{S})$, $148.9(\mathrm{C}=\mathrm{N}), 140.0,136.5,134.1,131.2,123.0,121.0$ ( phenyl ring). (Dhanya et al., 2014).
Compound (6). ${ }^{1} \mathrm{H}$ NMR (DMSO-d $\left.\mathrm{d}^{6}, 400 \mathrm{MHz}\right): \delta=$ $8.9(\mathrm{~s}, 1 \mathrm{H}, \mathrm{H}-\mathrm{C}=\mathrm{N}), 8.7(\mathrm{~s}, 1 \mathrm{H}, \mathrm{H}-\mathrm{C}=\mathrm{C}), 8.3-8.0(\mathrm{~m}$, 5H, Ar-H), 7.1-7.7 (m, 5H, Ar-H), $3.8\left(\mathrm{~s}, 3 \mathrm{H}, \mathrm{OCH}_{3}\right)$. ${ }^{13} \mathrm{C}$ NMR (DMSO-d $\left.{ }^{6}, 400 \mathrm{MHz}\right): \delta=160.1(\mathrm{C}=\mathrm{S})$, $150.3(\mathrm{C}=\mathrm{N}), 131,130,128,124,117,115$ (phenyl ring), $59\left(\mathrm{OCH}_{3}, 55(\mathrm{C}=\mathrm{C}-\mathrm{H})\right.$. (Dhanya et al., 2014).

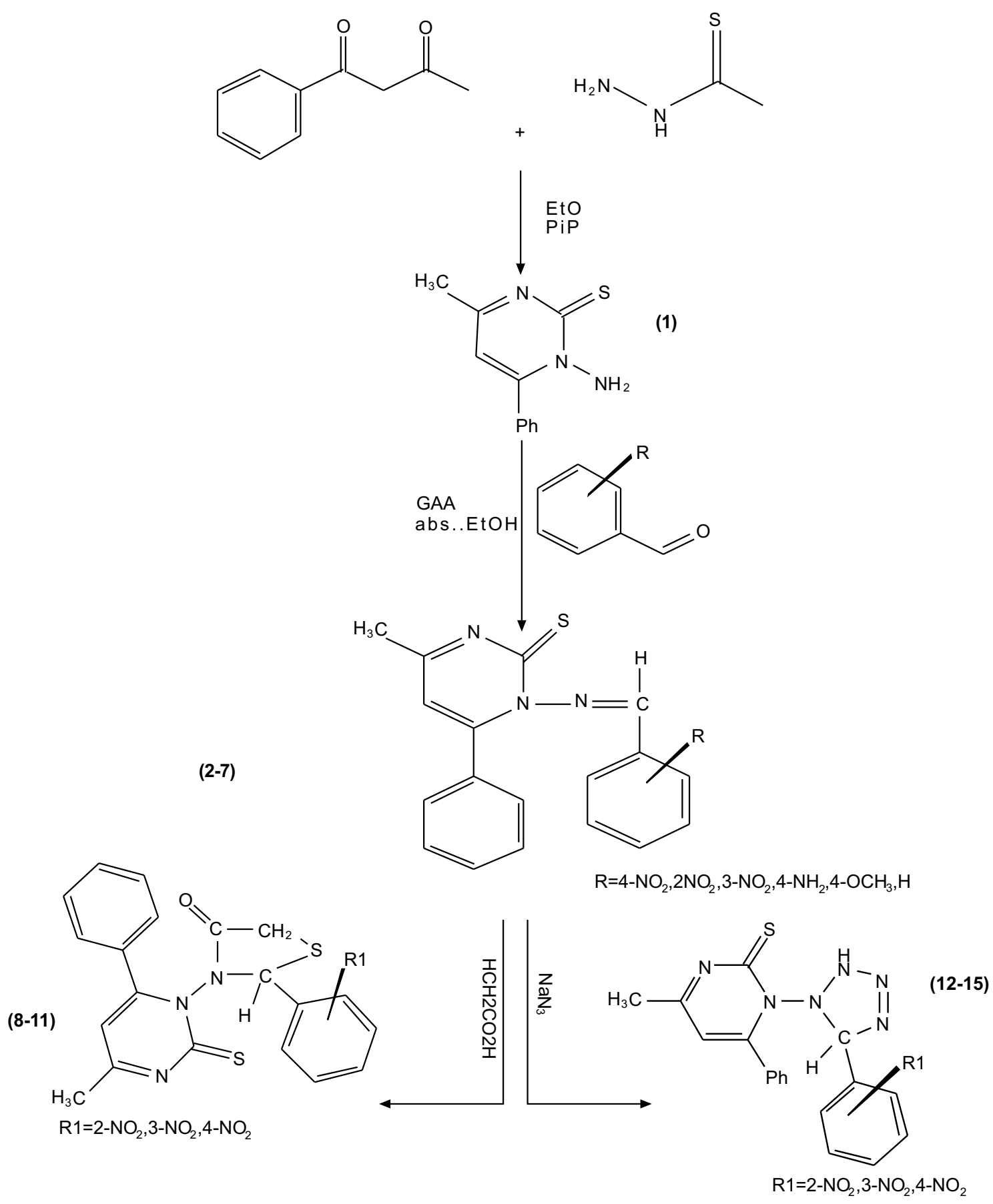

Scheme (1). Synthesis of compounds (1-15). 
Table 2. Some spectral data for compounds (1-15)

\begin{tabular}{|c|c|c|c|c|c|c|c|}
\hline \multirow{2}{*}{$\begin{array}{l}\text { Comp. } \\
\text { no. }\end{array}$} & \multicolumn{7}{|c|}{$\mathrm{V}\left(\mathrm{cm}^{-1}\right) \mathrm{IR}$} \\
\hline & $\mathrm{N}-\mathrm{H}$ tetrazole & $=\mathrm{C}-\mathrm{H}$ & $\mathrm{R}-\mathrm{CH}$ & $\mathrm{C}=\mathrm{N}$ Exo & $\mathrm{C}=\mathrm{S}$ & $\mathrm{N}-\mathrm{N}$ & Others \\
\hline 1 & $\ldots \ldots$ & 3198 & 2998 & 1598 Endo & 1081 & 936 & $3267-3401\left(\mathrm{NH}_{2}\right)$ \\
\hline 2 & $\ldots \ldots$ & 3145 & 2981 & 1596 & 1227 & 1062 & $\begin{array}{l}\mathrm{NO}_{2}(\text { Asy/sym }) \\
1521-1342\end{array}$ \\
\hline 3 & $\ldots \ldots$ & 3141 & 2977 & 1599 & 1221 & 1064 & $\begin{array}{l}\mathrm{NO}_{2}(\text { Asy/sym }) \\
1542-1346\end{array}$ \\
\hline 4 & $\ldots \ldots$ & 3089 & 2986 & 1580 & 1270 & 1085 & $\begin{array}{l}\mathrm{NO}_{2}(\text { Asy/sym }) \\
1513-1330\end{array}$ \\
\hline 5 & $\ldots \ldots$ & 3076 & 2963 & 1606 & 1214 & 1103 & $\left(\mathrm{NH}_{2}\right) 3215-3473$ \\
\hline 6 & $\ldots \ldots$ & 3113 & 2986 & 1585 & 1284 & 1084 & $(\mathrm{C}-\mathrm{O}-\mathrm{C}) 1118-1224$ \\
\hline 7 & $\ldots \ldots$ & 3195 & 2988 & 1590 & 1225 & 1079 & 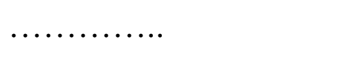 \\
\hline 8 & $\ldots \ldots$ & 3139 & 2975 & $\ldots \ldots$ & 1216 & 1064 & $\begin{array}{l}(\mathrm{C}=\mathrm{O}) \text { imide } 1700 \\
(\mathrm{C}-\mathrm{S}-\mathrm{C}) 810\end{array}$ \\
\hline 9 & $\ldots \ldots$ & 3073 & 2965 & $\ldots \ldots$ & 1198 & 1132 & $\begin{array}{l}(\mathrm{C}=\mathrm{O}) \text { imide } 1703 \\
(\mathrm{C}-\mathrm{S}-\mathrm{C}) 762\end{array}$ \\
\hline 10 & $\ldots \ldots$ & 3024 & 2982 & .. & 1207 & 933 & $\begin{array}{l}(\mathrm{C}=\mathrm{O}) \text { imide } 1704 \\
(\mathrm{C}-\mathrm{S}-\mathrm{C}) 809\end{array}$ \\
\hline 11 & $\ldots \ldots$ & $\begin{array}{l}3081- \\
3154\end{array}$ & $\begin{array}{l}2977- \\
2910\end{array}$ & $\ldots \ldots$ & $\begin{array}{l}1291- \\
1220\end{array}$ & $\begin{array}{l}995- \\
939\end{array}$ & $\begin{array}{l}(\mathrm{C}=\mathrm{O}) \text { imide } 1724,1652 \\
(\mathrm{C}-\mathrm{S}-\mathrm{C}) 757\end{array}$ \\
\hline 12 & 3356 & 3159 & 2962 & $\ldots \ldots$ & 1207 & 911 & $(\mathrm{~N}=\mathrm{N}) 1447$ \\
\hline 13 & 3423 & 3160 & 2966 & & 1211 & 935 & $(\mathrm{~N}=\mathrm{N}) 1418$ \\
\hline 14 & 3359 & 3088 & 2962 & $\ldots \ldots$ & 1174 & 926 & $(\mathrm{~N}=\mathrm{N}) 1448$ \\
\hline 15 & 3352 & 3192 & 2960 & & 1248 & 966 & $(\mathrm{~N}=\mathrm{N}) 1499,1441$ \\
\hline
\end{tabular}

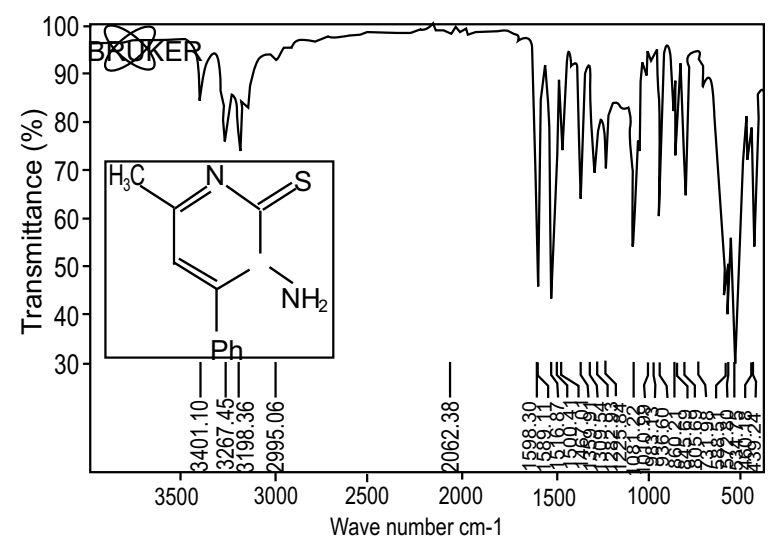

Fig. 1. FT-IR spectrum of compound (1).

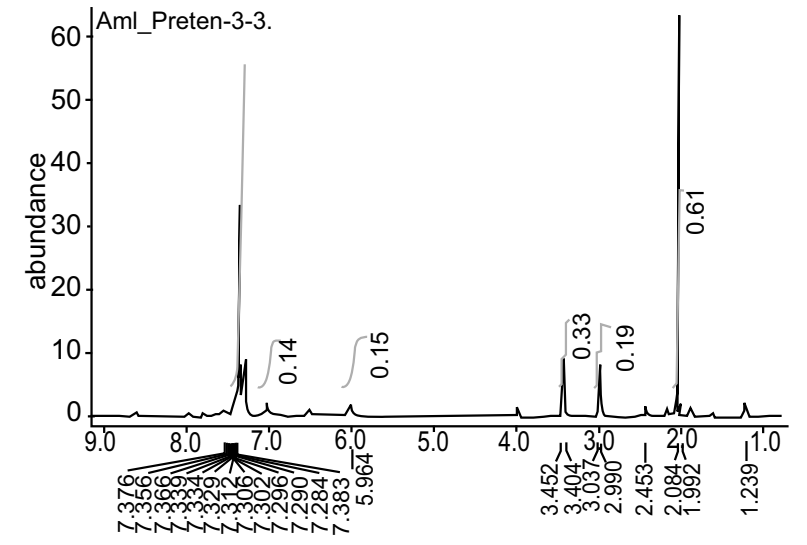

Fig. 2. ${ }^{1} \mathrm{H}-\mathrm{NMR}$ spectrum of compound (1). 
Compound (7). ${ }^{1} \mathrm{H}$ NMR (acetone- $\left.\mathrm{d}^{6}, 400 \mathrm{MHz}\right): \delta=$ $8.68(\mathrm{~s}, 1 \mathrm{H}, \mathrm{H}-\mathrm{C}=\mathrm{N}), 8.08-8.20(\mathrm{~m}, 4 \mathrm{H}, \mathrm{Ar}-\mathrm{H}), 7.55-$ 7.88 (m, 10H, Ar-H), 5.3 (s, 1H, C=C-H), 2.85 (br, 6H,

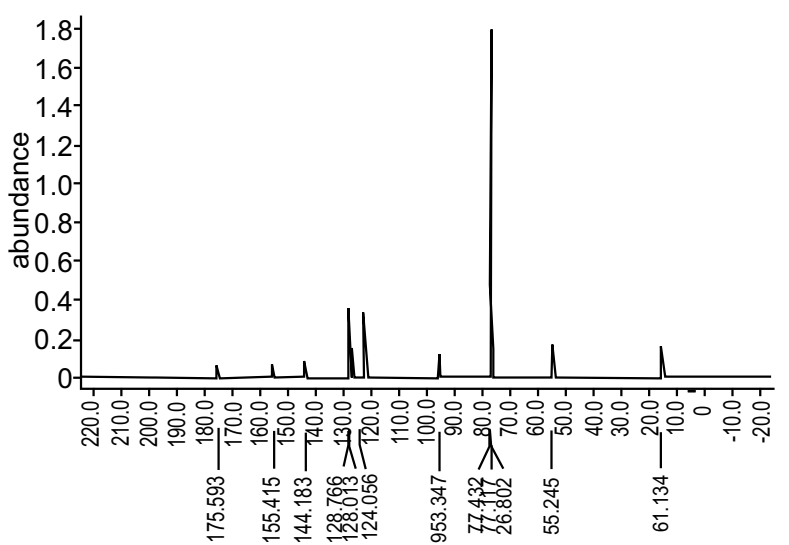

Fig. 3. ${ }^{13} \mathrm{C}-\mathrm{NMR}$ spectrum of compound (1).

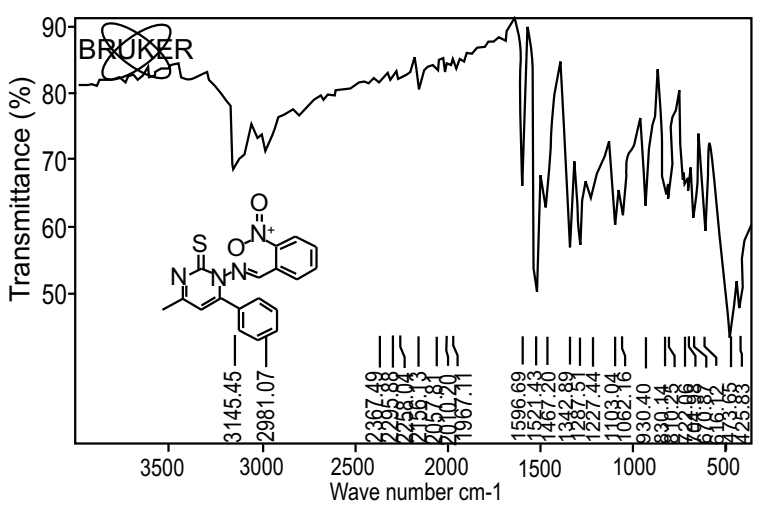

Fig. 4. FT-IR spectrum of compound (2).

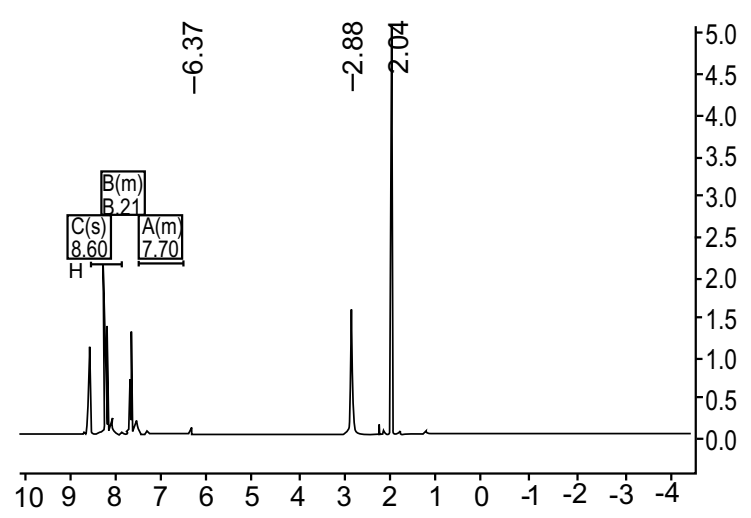

Fig. 5. ${ }^{1} \mathrm{H}-\mathrm{NMR}$ spectrum of compound (2). $\left.2 \mathrm{CH}_{3}\right) .{ }^{13} \mathrm{C}$ NMR (acetone $-\mathrm{d}^{6}, 400 \mathrm{MHz}$ ): $\delta=141.2$, 134.6, 133.1, 130.3, 128.8, 128.6, 124.8, 124.5,122.0 (phenyl ring), $29.5\left(\mathrm{CH}_{3}\right)$. (Dhanya et al., 2014).

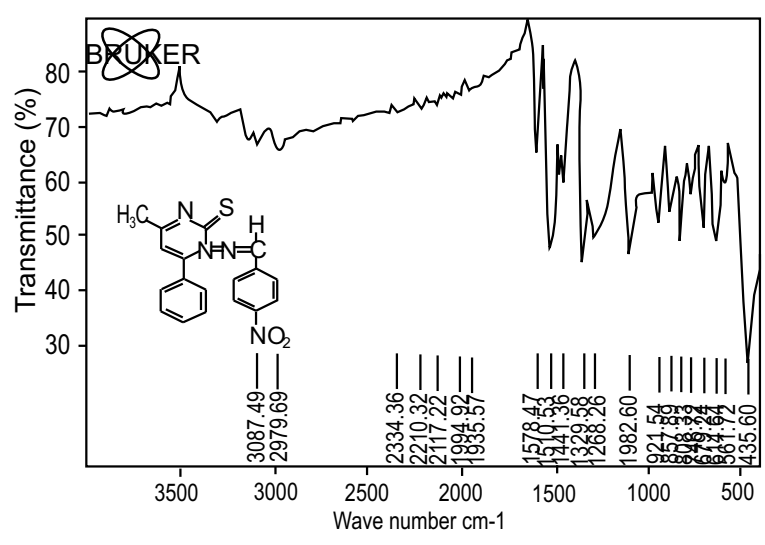

Fig. 6. FT-IR spectrum of compound (4).

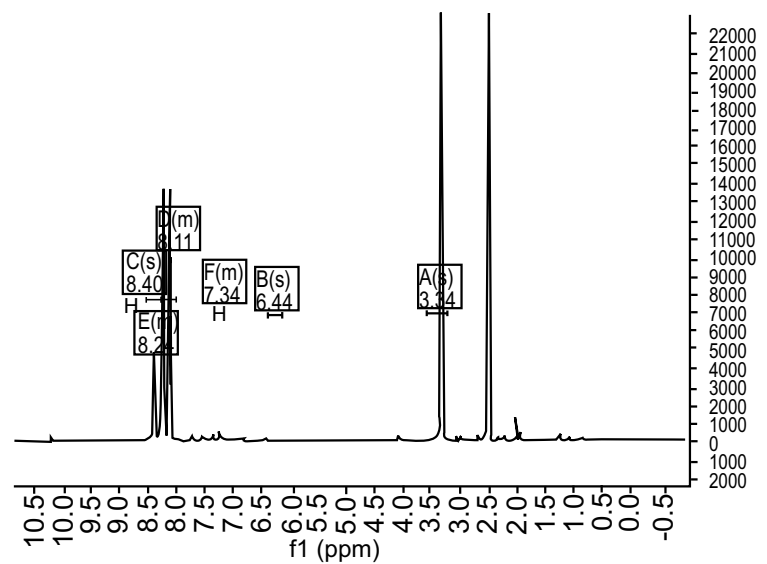

Fig. 7. ${ }^{1} \mathrm{H}-\mathrm{NMR}$ for compound (4).

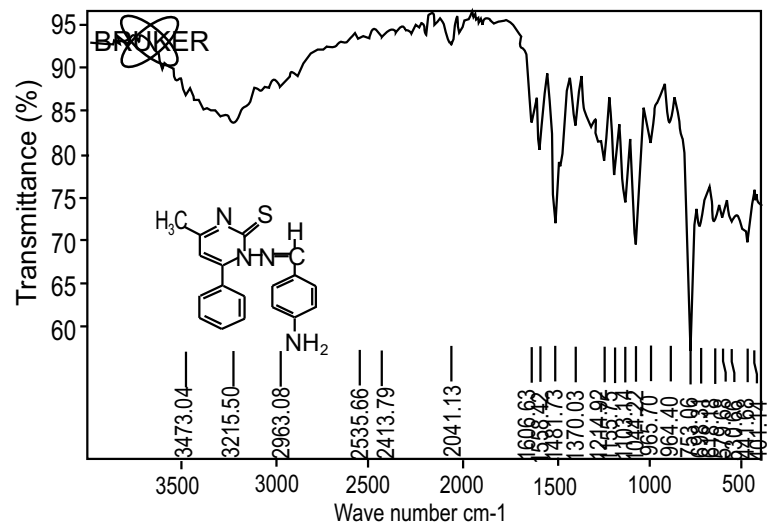

Fig. 8. FT-IR for compound (5). 


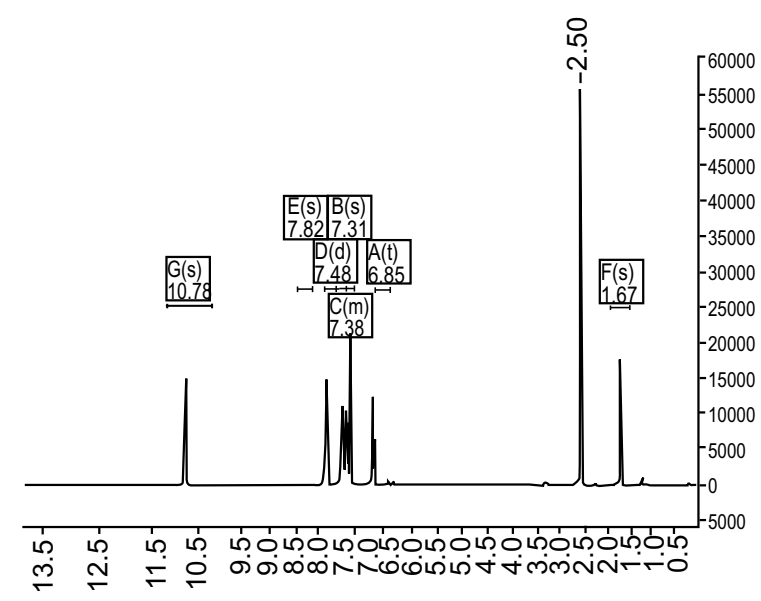

Fig. 9. ${ }^{1} \mathrm{H}-\mathrm{NMR}$ for compound (5).

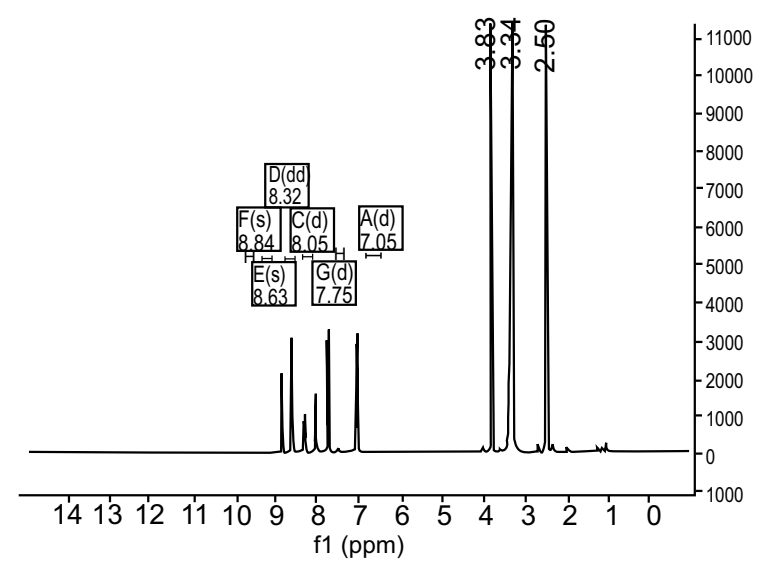

Fig. 10. ${ }^{1} \mathrm{H}-\mathrm{NMR}$ for compound (6).

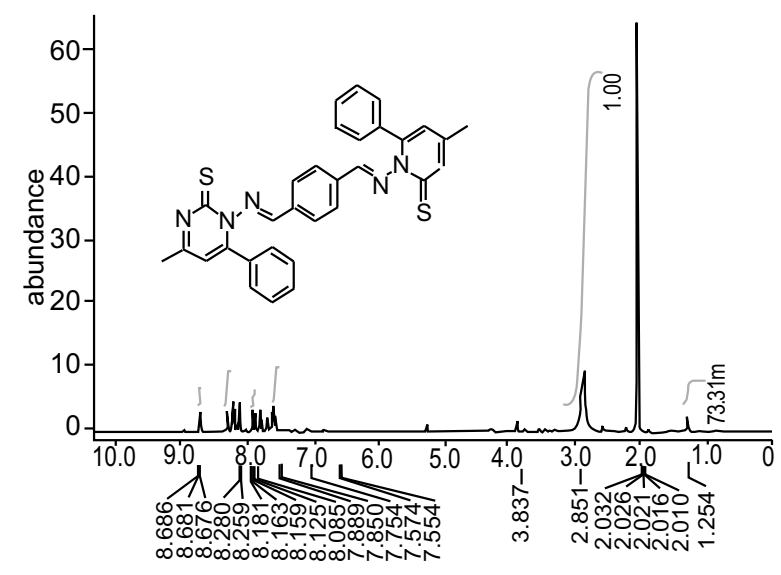

Fig. 11. ${ }^{1} \mathrm{H}$ - NMR spectrum of compound (7).

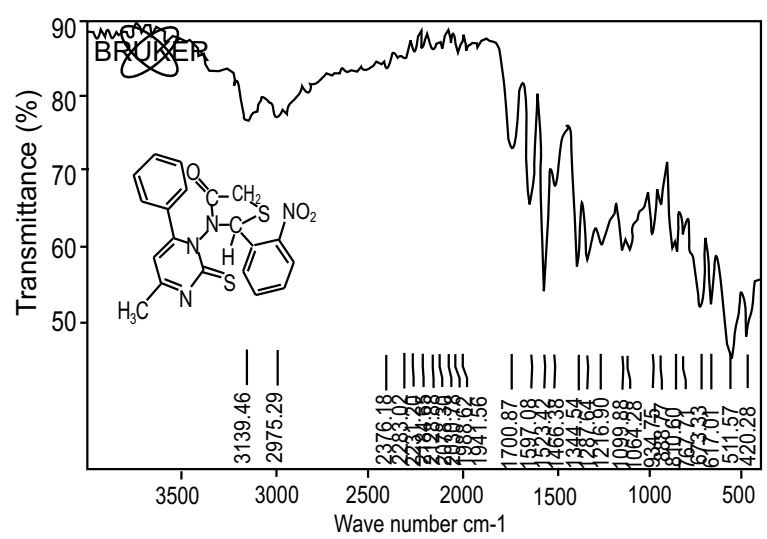

Fig. 12. FT-IR for compound (8).

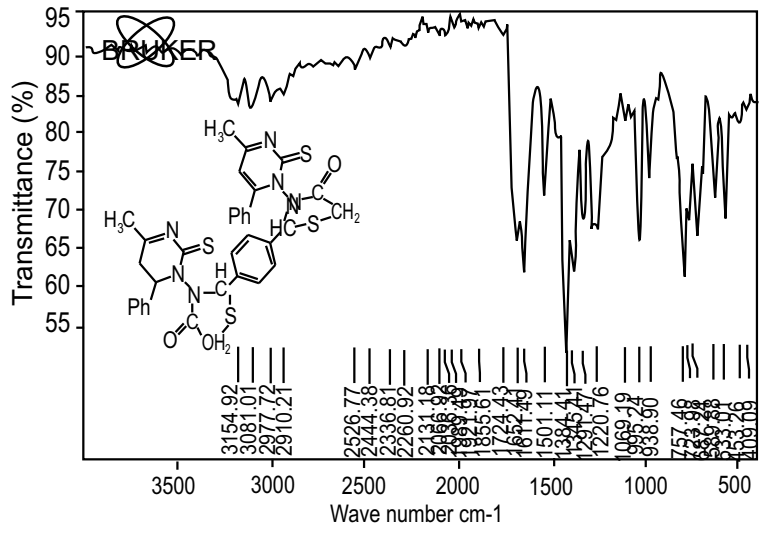

Fig. 13. FT-IR spectrum for compound (11).

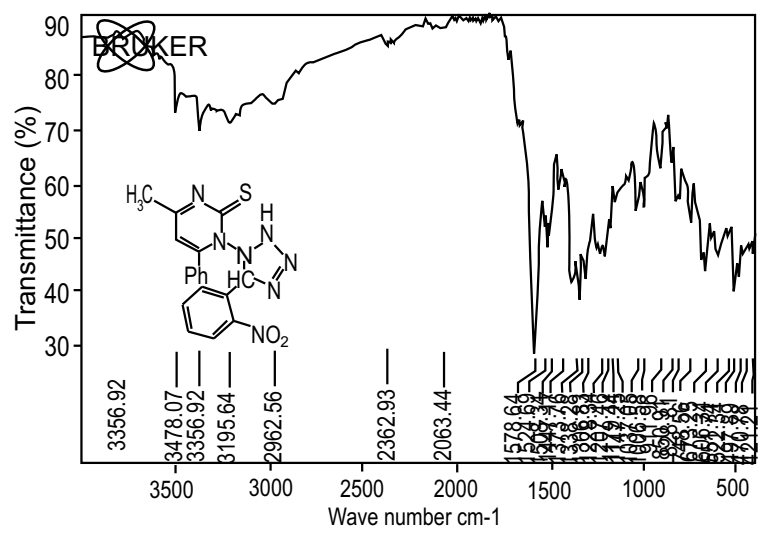

Fig. 14. FT-IR spectrum of compound (12).

Compound (10). ${ }^{1} \mathrm{H} \mathrm{NMR}\left(\mathrm{CDCl}_{3}, 400 \mathrm{MHz}\right): \delta=8.20$ - 8.26 (m,4H, Ar-H), 7.52-7.87 (m, 5H, Ar-H), 6,98, $(\mathrm{S}, 1 \mathrm{H}, \mathrm{H}-\mathrm{C}-\mathrm{N}), 5.77$ (s, 1H, H-C=C), 4.40 (br, 2H, $\mathrm{CH}_{2}$ ), 2.34 (s, 3H, $\mathrm{CH}_{3}$ ) (Kumar, 2012). 
Compound (12). ${ }^{1} \mathrm{H}$ NMR (DMSO-d $\left.\mathrm{d}^{6}, 400 \mathrm{MHz}\right): \delta=$ $11.6(\mathrm{~s}, 1 \mathrm{H}, \mathrm{NH}), 8.7$ (s, 1H, C=C-H), 8.1-8.4 (m, 4H, Ar-H), 7.2- 7.7 (m, 5H, Aar-H), 4.1 (s, 1H, H-C-N ). $3.4\left(\mathrm{~s}, 3 \mathrm{H}, \mathrm{CH}_{3}\right) .{ }^{13} \mathrm{C}$ NMR (DMSO-d 6 , 400MHz): $\delta=$ $179.1(\mathrm{C}=\mathrm{S}), 155(\mathrm{C}=\mathrm{N}), 149,140,135,134,131,129$, 128, 129, 124, 122, (phenyl ring), $95(\mathrm{C}=\mathrm{C}-\mathrm{H}), 55(\mathrm{H}-$ $\mathrm{C}-\mathrm{N}), 16\left(\mathrm{CH}_{3}\right)$ (Majeed and Saoud, 2013).

\section{Conclusion}

From the experiment it was concluded that the synthesis of tetrazole and thiazolidinones were prepared on safe and simplicity with a good product yield.

\section{Ankowledgement}

We are grateful to Department of Chemistry, College of Education for Pure Science, Mosul University, for the facilities given to perform this work. Thanks are also due to Pakistan Journal of Scientific and Industrial Research (PJSIR).

Conflict of Interest. The authors declare have no conflict of interest.

\section{Reference}

Al-Gwady, M.S., Mohammed, S.J., Sheat, A.M. 2018. Synthesis, characterisation and antimicrobial evaluation of some new heterocyclic compound using citric acid as a synthon. Pakistan Journal of Scientific and Industrial Research, 61: 66-73.

Al-Mosawi, S.K. 2014. Synthesis and characterization of heterocyclic schiff base, thaizolidione and chalcone as antibacterial agents. Research Journal of Pharmaceutical Biological and Chemical Sciences, 5: 411-417.

Dhanya, S., Ranjitha, C., Rama, M., Pai, K. 2014. Oxazepine derivative as an antitumor agent and snail1 inhibitor against human colorectal adenocarcinoma. International Journal of Innovative Research in Science Engineering and Technology, 3: $15357-15363$.

Harika, S., Sudha, B.N. 2014. Synthesis and characterization of N-thiadiazolyl thiazolidinone derivatives. International Journal of Research in Pharmacy and Science, 4: 13-16.

Hussain, Z., Yousif, E., Ahmed, A., Altaie, A. 2014. Synthesis and characterization of schiff's bases of sulfamethoxazole. Organic and Medicinal Chemistry Letters, 4: 1-4.
Kumar, K., Chandrashekar, K.B., Nagaraju, G., Madhu, G., Spoorthy, Y.N., Nath, L. 2012. Synthesis, characterization and biological evaluation of pyrazolones containing multi substituted thiazolidinones and oxadiazoles. Der Pharma Chemica, 4: 1603-1612.

Lakum, H.P., Shah, D.R., Chikhalia, K.H. 2014. Novel s- triaziny schiff base/chalcone congeners: rational, synthesis, antimicrobial and anti-TB evaluation. International Letters of Chemistry, Physics and Astronomy, 19: 56-73.

Mahmoud, M.J., Jassim, I.K., Mahmoud, M.A. 2013. Synthesis and characterization of five, seven heterocyclic membered rings. Baghdad Science Journal, 10: 803-817.

Majeed, I.Y., Al-Saady, D., Saoud, S.A. 2013. Synthesis and characterization of some new compounds derivatives from para-amino benzoic acid. International Journal for Sciences and Technology, 8: 6-11.

Moayed, M.E. 2017. Syntheses and spectral characterization of some pyrimidinones, pyrimidine thiones and 1,3-oxazepines, Ph.D. Thesis, in Chemistry, University of Mosul, Iraq.

Natiq, G.A., Hussein, Y., Al-Hashim. 2016. Preparation and characterization of some new schiff bases compounds with the study of biological effectiveness. International Journal of Current Research in Biosciences and Plant Biology, 3: 127-136.

Parekh, H.H., Parekh, K.A., Parekh, A.R. 2004. Synthesis of some 4-thiazolidinone derivatives as antitubercular agents. Journal of Science, Islamic Republic of Iran, 15: 143-148.

Patel, J.B., Desai, A. 2011. Synthesis of some new thiazolidinones derived from 1-p-tolylethanone and their antibacterial and antifungal activities. International Journal of Industrial Chemistry, 2: 4551.

Pradip, D., Berad, B.N. 2008. Synthesis characterization and antimicrobial study of substituted bis-[1,3,4]oxadizole, bis-[1,3,4]-thiadizole and bis-[1,2,4]triazole derivatves. Journal Indian Chemical Society, 85: 1153-1158.

Qien, G., Li, Cui, X., Hueng, J., Cui, Q.D., Zhang, R., Liu, F. 2006. Synthesis and herbicidal activities of fluorinecontaining 3-pyridylmethyl-2-phenyl iminothiazolidine derivatives. Journal of Fluorine Chemistry, 17: 182-186. 
Saad, M.M., Ismail, A.K. 2018. Preparation and identification of new azo-schiff base ligand (NASAR) and its divalent transition metal complexes. Journal of Pharmaceutical Sciences and Research, 10: 2175-2178.

Samadhiya, S., Halve, A. 2001. Synthetic utility of schiff bases as potential herbicidal agents. Oriental Journal of Chemistry, 17: 119-122.

Srivustava, S.K., Srivustava, S., Srivustava, S.D. 2002. Synthesis of 5-arylidene-2-aryl-3-(1,2,4-triazoloacetamidyl)-1,3-thiadiazol-4-ones as antibacterial, antifungal, analgesic and diuretic agents. Indian Journal of Chemistry, 41B: 1937-1945.

Selvam, T.P., James, C.R., Dniandev, P.V., Valzita, S.K. 2012. A mini review of pyrimidine and fused pyrimidine marketed drugs. Research in Pharmacy, 2: 1-9.

Subudhi, B.B., Panda, P.K., Tosh, B.K., Sahu, S., Majhi, P. 2005. Synthesis and biological activity evaluation of some azetidinone and thiazolidinone derivatives of coumarins. Dhaka University Journal of Pharmaceutical Science, 4: 87-92. 